\title{
THE CONVERSE OF A THEOREM OF TCHAPLYGIN ON DIFFERENTIAL INEQUALITIES
}

\author{
J. ERNEST WILKINS, JR.
}

1. Introduction. Suppose that $y(x)$ is a solution of the linear differential equation

$$
y^{\prime \prime}-p_{1} y^{\prime}-p_{2} y-q=0, \quad x \geqq x_{0},
$$

where $p_{1}(x), p_{2}(x)$ and $q(x)$ are continuous when $x \geqq x_{0}$, and that

$$
y\left(x_{0}\right)=y_{0}, \quad y^{\prime}\left(x_{0}\right)=y_{0}^{\prime} .
$$

Then if $v(x)$ satisfies the differential inequality

$$
v^{\prime \prime}-p_{1} v^{\prime}-p_{2} v-q>0,
$$$$
x \geqq x_{0},
$$

and the same boundary conditions as $y(x)$ at $x_{0}$, it is clear that the inequality

$$
v(x)>y(x)
$$

holds in some right-hand neighborhood of $x_{0}$. Tchaplygin ${ }^{1}$ has proved that the inequality (1.4) holds when $x_{0}<x \leqq x_{1}$ provided that there exists a solution $\lambda(x)$ of the Riccati equation

$$
\lambda^{\prime}+\lambda^{2}+p_{1} \lambda+\left(p_{1}^{\prime}-p_{2}\right)=0
$$

which is continuous when $x_{0}<x<x_{1}$. Let $X\left(x_{0}\right)$ be the least upper bound of values $x_{1}$ for which the Riccati equation admits a continuous solution when $x_{0}<x<x_{1}$. Then the inequality (1.4) holds when $x_{0}<x \leqq X\left(x_{0}\right)$, and Petrov [2] has shown that if $p_{1}$ and $p_{2}$ are constants no better result is true. That is, if $p_{1}$ and $p_{2}$ are constants and $X\left(x_{0}\right)$ is finite, then there exists a function $v(x)$ satisfying (1.3) and (1.2) for which $v(x)=y(x)$ at a point arbitrarily close to but greater than $X\left(x_{0}\right)$. It is the purpose of this paper to show that this last result is true without the restriction that $p_{1}$ and $p_{2}$ are constants. We prefer to state our results in terms of and make our proofs depend 1946.

Presented to the Society, November 2, 1946; received by the editors September 16,

${ }^{1}$ The author knows this result only by virtue of a reference to it contained in the paper of Petrov [2], and there it is not made clear whether or not Tchaplygin took the interval from $x_{0}$ to $x_{1}$ to be open, as we have written it, or closed or half-open. It follows, however, from the results obtained in $\$ 2$ that this statement is true for the open interval and hence is a fortiori true for the closed and half-open intervals. Numbers in brackets refer to the bibliography at the end of the paper. 
upon properties of solutions of the homogeneous linear differential equation

$$
u^{\prime \prime}-p_{1} u^{\prime}-p_{2} u=0,
$$

rather than solutions of the Riccati equation (1.5). Apart from the trivial advantage that we do not need to assume that $p_{1}(x)$ is differentiable, it is possible to give simpler and more natural proofs using (1.6) than (1.5).

2. The main theorem. We are going to prove the following theorem.

Theorem. If $y(x)$ satisfies (1.1) and (1.2), and if $v(x)$ satisfies (1.3) and (1.2), then the inequality (1.4) holds when $x_{0}<x \leqq x_{1}$ provided that there exists a solution $u(x)$ of (1.6) which does not vanish when $x_{0}<x<x_{1}$. Let $u_{0}(x)$ be a solution of (1.6) such that $u_{0}\left(x_{0}\right)=0, u_{0}^{\prime}\left(x_{0}\right)=1$, and let $X\left(x_{0}\right)$ be the first zero of $u_{0}(x)$ to the right of $x_{0}$ if any such zero exists; otherwise, let $X\left(x_{0}\right)=+\infty$. Then the interval where $x_{0}<x \leqq X\left(x_{0}\right)$ is the largest interval in which the inequality (1.4) can be asserted to hold. In other words, either $X\left(x_{0}\right)=+\infty$ or else there exists a function $v(x, k)$ for each sufficiently small positive $k$ which satisfies (1.3) and (1.2) and for which $v\left[X\left(x_{0}\right)+k, k\right]<y\left[X\left(x_{0}\right)+k\right]$.

Let us define $z(x)=v(x)-y(x)$. Then

$$
z^{\prime \prime}-p_{1} z^{\prime}-p_{2} z=\phi(x)>0, \quad z\left(x_{0}\right)=z^{\prime}\left(x_{0}\right)=0 .
$$

If $u(x)$ is a solution of (1.6) and one defines the Wronskian of $u(x)$ and $z(x)$ to be

$$
W(x)=u(x) z^{\prime}(x)-u^{\prime}(x) z(x),
$$

then it is easy to see that

$$
W^{\prime}(x)-p_{1}(x) W(x)=u(x) \phi(x), \quad W\left(x_{0}\right)=0,
$$

whence we have that

$$
W(x)=\int_{x_{0}}^{x} u(s) \phi(s) P(x, s) d s,
$$

where $P(x, s)$ is defined as

$$
P(x, s)=\exp \int_{s}^{x} p_{1}(t) d t .
$$

Suppose now that $z(x)$ vanishes at some point to the right of $x_{0}$, and that $x^{*}$ is the first such point. Then when $x=x^{*}$, equation (2.3) reduces to 


$$
u\left(x^{*}\right) z^{\prime}\left(x^{*}\right)=\int_{x_{0}}^{x^{*}} u(s) \phi(s) P\left(x^{*}, s\right) d s .
$$

Since $z(x)>0$ to the left of $x^{*}$ we have that $z^{\prime}\left(x^{*}\right) \leqq 0$. It now follows from equation (2.5) that $u(s)$ must change sign when $x_{0}<s<x^{*}$, for otherwise the two sides of $(2.5)$ could not have the same sign. This remark is equivalent to the first sentence of the theorem.

If $X\left(x_{0}\right)=+\infty$, then $z(x)$ can never vanish to the right of $x_{0}$. In this case the inequality (1.4) holds whenever $x>x_{0}$. Let us now suppose that $X\left(x_{0}\right)$ is finite. Let $x_{2}$ be any point to the right of $X\left(x_{0}\right)$ such that $u_{0}(x)$ has no zeros between $X\left(x_{0}\right)$ and $x_{2}$. Let $u(x)$ be the solution of (1.6) such that $u\left(x_{2}\right)=0, u^{\prime}\left(x_{2}\right)=-1$. It follows from the separation theorem for the zeros of solutions of second order linear homogeneous differential equations [1, p. 224] that there is a unique point $x_{1}$ between $x_{0}$ and $X\left(x_{0}\right)$ such that $u\left(x_{1}\right)=0$. For this function $u(x)$ we get from equation (2.3) that

$$
z\left(x_{2}\right)=\int_{x_{0}}^{x_{2}} u(s) \phi(s) P\left(x_{2}, s\right) d s .
$$

To complete the proof of the theorem it is sufficient to show that for each $x_{2}$ subject to the restrictions already imposed on it a positive function $\phi\left(s, x_{2}\right)$ can be found such that

$$
\int_{x_{0}}^{x_{2}} u(s) \phi\left(s, x_{2}\right) P\left(x_{2}, s\right) d s<0 .
$$

If $b>0$, the function $\phi\left(s, x_{2}\right)$ defined as

$$
\begin{array}{ll}
\phi\left(s, x_{2}\right)=[1-b u(s)] / P\left(x_{2}, s\right), & x_{0}<s<x_{1}, \\
\phi\left(s, x_{2}\right)=1 / P\left(x_{2}, s\right), & x_{1}<s<x_{2},
\end{array}
$$

is surely positive. For this function the integral (2.6) has the value

$$
\int_{x_{0}}^{x_{2}} u(s) d s-b \int_{x_{0}}^{x_{1}} u^{2}(s) d s
$$

and will certainly be negative if $b$ is sufficiently large.

To round out the results of this paper we shall now show that the number $X\left(x_{0}\right)$ defined in the theorem coincides with the number $X\left(x_{0}\right)$ defined in the introduction by means of the Riccati equation (1.5). We prefer to replace (1.5) by

$$
\mu^{\prime}+\mu^{2}-p_{1} \mu-p_{2}=0,
$$


where $\mu=\lambda+p_{1}$, and define $X_{1}\left(x_{0}\right)$ as the least upper bound of numbers $x_{1}$ such that the equation (2.7) admits a solution continuous when $x_{0}<x<x_{1}$. Since $p_{1}$ is continuous this number is the same as that defined for the equation (1.5) in case $p_{1}$ is differentiable, but $X_{1}\left(x_{0}\right)$ is defined even though $p_{1}$ is not differentiable. A function $\mu(x)$ satisfies (2.7) if and only if $\mu(x)=u^{\prime}(x) / u(x)$, where $u(x)$ is a nontrivial solution of (1.6). Hence solutions $\mu(x)$ of (2.7) which are continuous when $x_{0}<x<x_{1}$ correspond in a one-to-one fashion with families of functions $c u(x)$, where $c$ is an arbitrary nonzero constant, which satisfy (1.6) and which do not vanish when $x_{0}<x<x_{1}$. It follows that $X_{1}\left(x_{0}\right)$ is the least upper bound of values $x_{1}$ for which the equation (1.6) admits a solution not vanishing when $x_{0}<x<x_{1}$. Since every solution of (1.6) must vanish when $x_{0}<x \leqq X\left(x_{0}\right)$ we have that $X_{1}\left(x_{0}\right) \leqq X\left(x_{0}\right)$, and since $u_{0}(x)$ does not vanish when $x_{0}<x<X\left(x_{0}\right)$ we have that $X\left(x_{0}\right) \leqq X_{1}\left(x_{0}\right)$. Therefore, $X\left(x_{0}\right)=X_{1}\left(x_{0}\right)$.

\section{BibliograPHY}

1. E. L. Ince, Ordinary differential equations, Dover, New York, 1944.

2. V. N. Petrov, The limits of applicability of $S$. Tchaplygin's theorem on differential inequalities to linear equations with usual derivatives of the second order, C. R. (Doklady) Acad. Sci. URSS. vol. 51 (1946) pp. 255-258.

American Optical Company 